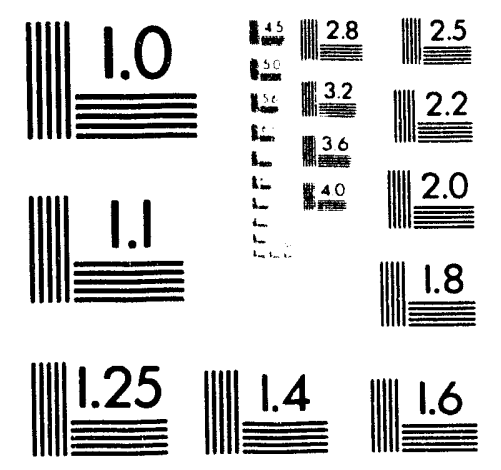



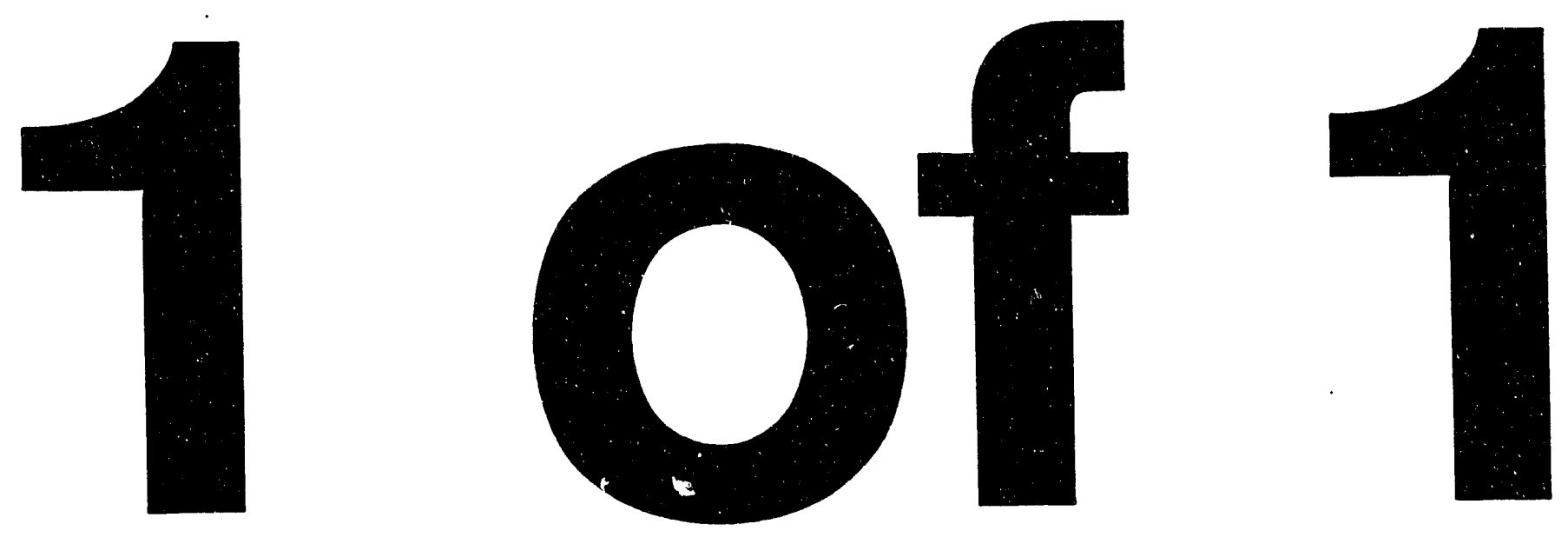


\title{
$\operatorname{Coy} 4936248-2$
}

\section{Forward Scattering of Neutrons from \\ Polymeric and Magnetic Multilayers}

\author{
G. P. Felcher
}

Materials Science Division

Argonne National Laboratory

$\therefore$ Argonne, IL 60439
RECEIV:-

SEP 271993

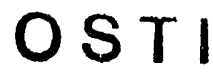

\begin{abstract}
The submitled manuscript has been authored by a
contractor of the U.S. Government under contract No. W-31-109-ENG-38. Accordingly, the U.S. Government retains a nonexclusive, royalty-free license to publish or reproduce the published form of this contribution, or allow others to do so, for U.S. Government purposes.
\end{abstract}

August, 1993

To be published in Physica B

\section{DISCLAIMER}

$/ \mathrm{sm}$

\begin{abstract}
This report was prepared as an account of work sponsored by an agency of the United States Government. Neither the United States Government nor any agency thereof, nor any of their employees, makes any warranty, express or implied, or assumes any legal liability or responsibility for the accuracy, completeness, or usefulness of any information, apparatus, product, or process disclosed, or represents that its use would not infringe privately owned rights. Reference herein to any specific commercial product, process, or service by trade name, trademark, manufacturer, or otherwise does not necessarily constitute or imply its endorsement, recommendation, or favoring by the United States Government or any agency thereof. The views and opinions of authors expressed herein do not necessarily state or reflect those of the United States Government or any agency thereof.
\end{abstract}

INVITED PAPER - 3rd International Conference on Surface X-Ray and Neutron Scattering, Dubna, Russia, June 24, 1993

This work is supported by the Division of Materials Sciences, Office of Basic Energy Sciences of DOE, under Contract No. W-31-109-ENG-38. 
Forward scattering of neutrons from polymeric and magnetic multilayers.

\author{
G.P. Felcher \\ Argonne National Laboratory, Argonne IL 60439
}

\begin{abstract}
Grazing incidence neutrons are not only reflected and refracted from imperfect layers, but also partially scattered by lateral dishomogeneities. In general, scattering may take place both in the reflection plane ("forward scattering") and out of it. The forward scattering from multilayers is highly structured in ridges, whose maxima can be indexed in terms of the multilayer spacings. In experiments on diverse diblock copolymers two kind of ridges were found, either at constant $-k_{z}$ loci or else at constant $-q_{z}$ loci. The relative intensity of the ridges appears to be related to the type and size of imperfections of the lamellar structure. Constant $-\mathrm{q}_{\mathrm{z}}$ streaks of magnetic nature were also found in the forward scattering of metallic superlattices ( $\mathrm{Fe} / \mathrm{Cr}, \mathrm{Co} / \mathrm{Ru}, \mathrm{Fe} / \mathrm{Nb}$ ) in the antiferromagnetic state. Here the diffuse scattering appears around the antiferromagnetic peaks while absent from the structural peaks, as if the crystalline superlattice were to partition into antiferromagnetic domains of limited lateral extension and columnar character.
\end{abstract}




\section{Introduction.}

In grazi. s $_{5}$ incidence geometry neutrons are either refracted or specularly reflected from an exactly planar sample. At an angle of incidence $\theta_{\mathrm{i}}$, and for a neutron wavelength $\lambda$, the component of the incidei't wavevector perpendicular to ther surface is $k_{z i}=2 \pi$ $\sin \theta_{\mathrm{i}} / \lambda$. Similarly for the exit beam $\mathrm{k}_{\mathrm{zf}}=2 \pi \sin \theta_{\mathrm{f}} / \lambda$. The scattering vector is simply $\mathrm{q}_{\mathrm{z}}=\mathrm{k}_{\mathrm{zi}}+\mathrm{k}_{\mathrm{zf}}$. From the intensity reflected as a function of $\mathrm{q}_{\mathrm{z}}$ the depth profile of the material may be obtained with great accuracy[1,2]. When the sample's planes are not perfect (because the surface or some interfaces are rough, or the layers are undulated, or the layers are marred by impurities) neutrons are also scattered: the scattering may take place in the plane of reflection (defined by the angle of incidence $\theta_{\mathrm{i}}$ and reflection $\theta_{\mathrm{f}}$ ), or else at an angle $\Delta \phi$ out of the reflection plane. By applying the conditions of conservation of energy and momentum it is easy to see $[3,4]$ that in the former type of scattering objects up to a size of the order of several microns can be observed. These objects are two orders of magnitude larger than those observable in other small angle scattering geometries. The present note is concerned with the phenomenology of this kind of scattering - which shall be named forward scattering - from imperfect multilayers.

The data presented here were collected at two POSY reflectometers[5] at the Intense Pulsed Neutron Source at Argonne National Laboratory. In both instrument a pulsed beam of neutrons, of all wavelengths in the thermal range, are brought onito the sample at an angle $\theta_{i}$. Position sensitive detectors record the neutrons reflected at $\theta_{\mathrm{f}}=\theta_{\mathrm{i}}$ (as well as the scattered neutrons at $\theta_{\mathrm{f}} \neq \theta_{\mathrm{i}}$ ) as a function of their time-of-flight from the source, and thus of their wavelength. The detectors are position-sensitive only along one direction (increasing $\theta$ ) and in practice integrate over $\Delta \phi$. Measurements were performed on polymers at the highly automated instrument POSY II. Magnetic multilayers were studied at the polarized neutrons reflectometer POSY I.

\section{Polymeric multilayers.}

Fig.1 shows the forward scattering from a film made of symmetric, diblock copolymers of polystyrene (PS) and polymethylmethacrylate (PMMA), for short P(S-bMMA). Thin films of P(S-b-MMA), cast onto silicon substrates, heated to temperatures above the glass transition temperature $\left(\sim 100^{\circ} \mathrm{C}\right)$, microphase separate into a lamellar morphology [6,7]. The lamellae are parallel to the film surface, and their thickness is given by $(n+1 / 2) L$ where $n$ is an integer and $L$ is the periodicity of the layers ( $n$ this sample of molecular weight $\sim 10^{5}, \mathrm{~L}=520 \AA$ ). PMMA segregates to the substrate and PS to the air surface. The sample whose scattering is presented in Fig. 1 had a thickness of $3.4 \times 10^{3} \AA$ 
which corresponds to an integer number of layers $(n=6)$ and thus, after annealing, did not show the islands or wells that form at the surface for an arbitrary film thickness[6]. However, a strong perturbation was introduced into the layering by mixing into $P(S-b$ MMA) $10 \%$ of pure PMMA $\left(M_{W}=5 \times 10^{4}\right)$. Since PS and PMMA are not miscible, the homopolymer is expected to share the same volume of the PMMA portion of the copolymer. In order to enhance the optical contrast of the lamellae, hydrogen was substituted with deuterium in the PS block of the copolymer.

Fig. 1 was obtained for an angle of incidence $\theta_{i}=1.58^{\circ}$. The abscissa is given in terms of the angle of scattering $\theta_{\mathrm{i}}+\theta_{\mathrm{f}}$ and the ordinate as the neutron wavelength. Although such representation may appear clumsy and tog linked to the geometry of the experiment, it is actually more readable than, for instance, a representation in the $\mathbf{q}$ space ( $\mathbf{q}$ is the momentum transfer in vacuum, with coordinates $\mathrm{q}_{\mathrm{z}}, \mathrm{q}_{\mathrm{x}}$ ). Well visible is the reflection line at $\theta_{\mathrm{i}}=\theta_{\mathrm{f}}$. The angle $\theta_{\mathrm{f}}=0$ corresponds to the surface horizon, which divides the neutrons scattered back in free space from those refracted into the substrate and exiting from its edge. As in a Laue camera, here the whole scattering spectrum is recorded: no features are missed, as may be the case when a single wavelength is used or a detector with a single position channel. Fig. 2 shows the position of the local maxima obtained at each wavelength, just for the neutrons scattered above the horizon. In Fig.2 are presented as well the loci of the maxima from runs at $\theta_{i}=0.6^{\circ}$ and $1.05^{\circ}$. All the datapoints fall on simple patterns, to be interpreted with the help of the specular reflectivity [8].

The specularly reflected intensity is presented in the insert of Fig.2. Maxima are visible at the critical momentum $\left(\mathrm{q}_{z c}=0.012 \AA^{-1}\right)$ and at $\mathrm{q}_{z .1}=0.0208, \mathrm{q}_{z .2}=0.0336$ and $q_{z 3}=0.0468 \AA^{-1}$ (the difference between adjacent maxima, $\Delta q_{z} \sim 2 \pi / L$, where $L$ is the periodicity of the layer). The loci of the diffuse scattering maxima obey the simple relation

$2 \pi \sin \theta_{f} / \lambda=q_{z M} / 2=k_{z M}$

where $M$ takes one of the values given above. Eq.(1) gives rise to the sets of straight lines ending up, for $\lambda=0$, to $\theta_{i}$ : clearly they are loci of the majority of datapoints. The constant$\mathrm{k}_{\mathrm{z}}$ scattering was first discovered long time ago for $\mathrm{M}=\mathrm{c}$ by Yoneda [9](for symmetry, $\mathrm{k}_{\mathrm{z}}$ can be the exit or the entrance beam), and recently "superYoneda", or constant- $k_{z M}$ scattering, has been observed for values of $M$ indicating one of the interference naxima of the reflectivity [3]. The position of the ridges is independent of the angle of incidence $\theta_{i}$; in other words, the memory of $\theta_{i}$ is lost and the indexing is done in terms of the wavevector of the exiting wave $k_{z f}$ only. The process may be described in the following way. Suppose that lateral imperfections scatter the incident plane wave into spherical waves; these may be decomposed in plane wave components $k_{z i}$ which, for the special 
values of $\mathrm{q}_{z \mathrm{M}}$ given above, are reflected by the laterally averaged sample. The relative $\mathrm{k}_{z M}$ may be considered as open channels into which the scattered neutrons are allowed to escape. In this pictorial description the constant- $\mathrm{k}_{7}$ scattering results from the sequence of two events, the first the incoherent scattering from lateral imperfections, the second the specular reflectivity from the mean lamellar structure.

Some of the datapoints do not fit along the constant $-\mathrm{k}_{\mathrm{z}}$ lines, but instead are aligned along a straight line common to all scattering spectra regardless of the angle of incidence. This line is described by

$\mathrm{k}_{\mathrm{zi}}+\mathrm{k}_{\mathrm{zf}}=\mathrm{q}_{\mathrm{z} 3}$

The constant- $\mathrm{q}_{z}$ scattering has also been observed by many authors $[3,10-12]$ and it has been interpreted in terms of a "conformal roughness". Basically in this model the multilayer is formed by a sequence of interfaces in registry to each other but not with a reference plane: the multilayer, rather than planar, is wavy or corrugated. In the extremal case the roughness is conformal over the entire thickness of the film, and then the intensity of the scattered radiation at fixed $\mathrm{q}_{x} \neq 0$ has exactly the same $\mathrm{q}_{z}$ dependence of the specularly reflected pattern, up to and including the oscillations due to the total film thickness. If the roughness is conformal over only part of the sample's thickness this correspondence gradually is lost, starting from the total thickness oscillations. In the P(S-b-MMA) sample with layers distorted by the addition of PMMA not much conformality is expected nor observed. What is observed is that constant $-\mathrm{k}_{\mathrm{z}}$ and constant $-\mathrm{q}_{\mathrm{z}}$ types of ridges coexist. At their intersection, band structure effects are visible, indicating that some kind of interaction takes place between different modes. Only one constant $-\mathrm{q}_{z}$ ridge is clearly marked. In other samples, instead, the diffuse scattering is preponderantly along constant $\mathrm{q}_{z}$ lines, as it will be shown in an example drawn from research on magnetic samples.

\section{Magnetic multilayers}

Magnetic multilayers are made of a periodic sequence of couples, formed by a layer of ferromagnetic metal ( $\mathrm{Fe}, \mathrm{Co}, \mathrm{Ni}$ or a rare earth) a few tens of Angstroms thick, followed by a layer of non magnetic metal, or spacer, of a given thickness. First for very selected couples, then for a rapidly expanding host of combinations it was found that the coupling between subsequent ferromagnetic layers oscillates from ferromagnetic to antiferromagnetic to ferromagnetic again as the thickness of the non-magnetic spacers is increased[13-15]. The nature of the coupling, inferred from the magnetization measurements, was first directly observed by neutron reflection. In all recorded cases the alignment of the magnetization of the subsequent layer was either ferrromagnetic (F) or antiferromagnetic 
(AF) of the type +-+-, with a simple doubling of the chemical periodicity. The AF state can be suppressed by a saturating magnetic field. Examples of magnetic multilayers studied by neutron reflection comprise $\mathrm{Fe} / \mathrm{Cr}, \mathrm{Co} / \mathrm{Ru}, \mathrm{Ni} / \mathrm{Ag}, \mathrm{Co} / \mathrm{Cu}$ and $\mathrm{Fe} / \mathrm{Nb}[16]$.

Sizeable forward scattering has been almost universally observed in sputtered samples of magnetic multilayers in the AF state [17]. In Fig. 3 is shown the intensity contour map of a Fe/Cr multilayers [18] in the $\mathrm{q}_{x}, \mathrm{q}_{z}$ plane. The forward scattering has the form of a ridge of constant $-\mathrm{q}_{\mathrm{z}}$ centered around the $\mathrm{AF}$ peak. In contrast, at the first Bragg reflection of the modulated chemical structure no diffuse components are present. The forward scattering is of magnetic origin, as if the antiferromagnetic domains had finite size. To describe the magnetic configuration is, sufficient to think the multilayer as divided in columnar domains The sublattice magnetization of each domain is totally defined by the orientation of the magnetic moments in the surface layer. In absence of an external magnetic field, these moments are likey to point along one of the equivalent crystallographic axes.

The lateral dimensions of the domains observed for $\mathrm{Fe} / \mathrm{Cr}$ can be calculated with the help a simple formula. In the kinematic approximation the diffuse intensity around the antiferromagnetic peak is [16]:

$$
J_{x} \cdot J_{z}=\frac{\sin ^{2}\left(N_{z} a_{z} q_{z} / 2\right)}{\sin ^{2}\left(a_{z} q_{z} / 2\right)} \cdot \frac{\sin ^{2}\left(N_{x} a_{x} q_{x} / 2\right)}{\sin ^{2}\left(a_{x} q_{x} / 2\right)}
$$

Fluctuations along $y$ were omitted. In Eq.(3), a is the antiferromagnetic spacing and $N_{z}$ is the number of layers composing the film. $a_{x}$ is a dummy parameter and what is of interest in the $x$ direction is the total length $L_{x}=N_{x} a_{x}$. At the Bragg reflection $q_{x}=0$ and the arguments in $J_{z}$ are multiples of $2 \pi$. When the incident wavevector $k=2 \pi / \lambda$ is changed (but the angle of incidence $\theta_{i}$ remains constant) the maximum of $J_{z}$ occurs always for the same value of $\mathrm{q}_{z}$. Under this condition the exit beam makes with a surface an angle $\theta_{\mathrm{f}} \neq \theta_{\mathrm{i}}$ and $\mathrm{q}_{\mathrm{x}}$ becomes finite. At the onset of $\mathrm{q}_{\mathrm{x}}, \mathrm{J}_{\mathrm{x}}$ rapidly decreases; $\mathrm{L}_{\mathrm{x}}$ may be chosen by finding the value of $\mathrm{q}_{\mathrm{x}}$ at which $\mathrm{J}_{\mathrm{x}}=0$. For instance, for the sample of $\mathrm{Fe} / \mathrm{Cr}$ giving rise to the scattering of Fig. $3, \mathrm{~L}_{\mathrm{x}} \sim 0.4 \mu \mathrm{m}$.

\section{Discussion}

The diffuse scattering of magnetic multilayers is characterized by a constant- $\mathrm{q}_{\mathrm{z}}$ ridge and yet is not due to conformal roughness. Drawing an analogy with conventional crystallography, conformal roughness merely distorts a single crystal; instead the antiferromagnet is composed of a collage of crystallites. It would be interesting to ascertain the legitimacy of the multidomain model from the detailed $\mathrm{q}_{\mathrm{x}}$ dependence of the scattered 
intensity. However, such an analysis would require magnetic multilayers in which the distribution of domains were more uniform than in the present sample.

In summary, some aspects were shown of the forward scattering of imperfect multilayers. From the time of Yoneda's observations a robust work of development has taken place[19-21] to explain the features of forward scattering in terms of the distorted wave Born approximation For multilayers such approach might be unwieldy except for a few model systems. In an attempt to synthesize the main features of the forward scattering [22], contour plots were presented both in in $\mathrm{q}_{\mathrm{x}}, \mathrm{q}_{\mathrm{z}}$; and $\theta, \lambda$; coordinates. In the same scale was presented the topology of the maxima of the forward scattered neutrons. This last display may turn out to be a 'useful board to test both the approximations to the scattering theory and the model structural imperfections to which link the observations.

Acknowledgment. This work was supported by the US Depatment of Energy, BES-MS under contract No. W-31-109-ENG-38. 


\section{RIFERENCES}

1. J. Penfold and R.K. Thomas, J. Phys. C 2, 1369 (1990)

2. T.P. Russell,Mat. Sci. Reports 5,171 (1990)

3. S.K. Sinha, Physica B, 173, 25 (1991)

4. M. Tolan, G. König, L. Brügemann, W. Press, F.B. Brinkop and J.P. Kotthaus, Europhysics Lett., 20,223 (1992)

5. A. Karim, B.H. Arendt, R.Goyette, Y.Y. Huang, R. Kleb and G.P. Felcher, Physica B173, 17 (1991)

6. G. Coulon, T.P. Russell, V.R. Deline and P.F. Green, Macromolecules, 22, 2581 (1989)

7. S.H. Anastasiadis, T.P. Russell, S.K. Satija, and C.F. Majkrzak, Phys. Rev. Lett. 62, 1852 (1989); J. Chem. Phys., 92, 5677 (1990)

8. G.P. Felcher, R.J. Goyette, S. Anastasiadis, T.P. Russell, M. FOster and F. Bates, submitted, Phys. Rev. Lett.

9. Y. Yoneda, Phys.Rev. 131, 2010 (1963)

10. D.G. Stearns, J. Appl. Phys. 71, 4286 (1992)

11. D. Bahr, W. Press, R. Jebasinski and S. Mantl, Phys. Rev. B47, 4385 (1993)

12. Y.H. Phang, D.E. Savage, R. Kariotis and M. G. Lagally, J. Appl. Phys. (to be published)

13. P. Gruenberg, R. Schreiner, Y. Pang, M.B. Brodsky and H. Sowers, Phys. Rev. Lett. 57, 2442 (1986)

14. M.N. Baibich, J.M. Broto, A. Fert, F. Nguyen van Dau, F. Petroff, P. Etienne, G. Creuzet and A. Friederich, Phys. Rev. Lett. 61, 2472 (1988)

15. S.S.P. Parkin, N. More and K.P. Roche, Phys, Rev. Lett. 64, 2304 (1990)

16. G.P. Felcher, Physica B (to be published)

17. Y.Y. Huang, G.P. Felcher and S.S.P. Parkin, Journal of Magn. and Magn. Mat., 99, L31, (1991)

18. W. Hahn, M. Löwenhaupt, Y.Y. Huang, G.P. Felcher and S.S.P. Parkin, submitted, Journ. Appl. Phys.

19. A. Steyerl, Z.Phys. 254, 169 (1972)

20. S. Garoff, E.B. Sirota, S.K. Sinha and H.B. Stanley, Phys. Rev. B38, 2297 (1988)

21. R. Pynn, Phys. Rev. B45, 602 (1992)

22. J.B. Kortright, J. Appl. Phys. 70, 3620 (1991) 


\section{FIGURE CAPTIONS}

Fig. 1. Contour plot (on a $\log$ scale) of the intensities forwardly scattered from a sample of PS-PMMA copolymer containing 10\% PMMA homolymer. The angle of incidence is $\theta_{\mathrm{i}}=1.58$ degrees. The abscissa is $\theta_{\mathrm{i}}+\theta_{\mathrm{f}}: \theta_{\mathrm{f}}=0$ corresponds to the horizon of the sample surface; below this angle the neutrons are refracted from the sample, and exit from the edge of the substrate. The specularly reflected beam is located at $\theta_{\mathrm{i}}=\theta_{\mathrm{f}}$.

Fig.2 Lori of the scattering maximaifor the sample described in Fig.1, at three different angles of incidence (for clarity, maxima relative to the specularly reflected beams have been omitted). In the chosen axes the constant- $k_{z}$ lines become $\left[\left(\theta_{i}+\theta_{\mathrm{f}}\right) / q_{z M}\right]-$ $\left.\theta_{\mathrm{i}} / \mathrm{q}_{\mathrm{zM}}\right]=\lambda / 4 \pi$. Constant $-\mathrm{q}_{\mathrm{z}}$ lines become $\left(\theta_{\mathrm{i}}+\theta_{\mathrm{f}}\right) / \mathrm{q}_{\mathrm{zM}}=\lambda / 2 \pi$. In the insert: the reflectivity of the same sample (multiplied by $\mathrm{q}_{\mathrm{z}}{ }^{4}$ ).

Fig.3 a) Contour plot of the neutron intensities forwardly scattered from a sputtered multilayer of Fe/Cr. The thickness of the Fe layers is $30 \AA$, that of $\mathrm{Cr} 10 \AA$. The magnetic structure is antiferromagnetic, and it gives rise to the intensity scattered at $\mathrm{q}_{\mathrm{z}} \sim 0.075 \AA^{-1}$. At twice this value is visible the Bragg reflection due to the modulation in material composition. 


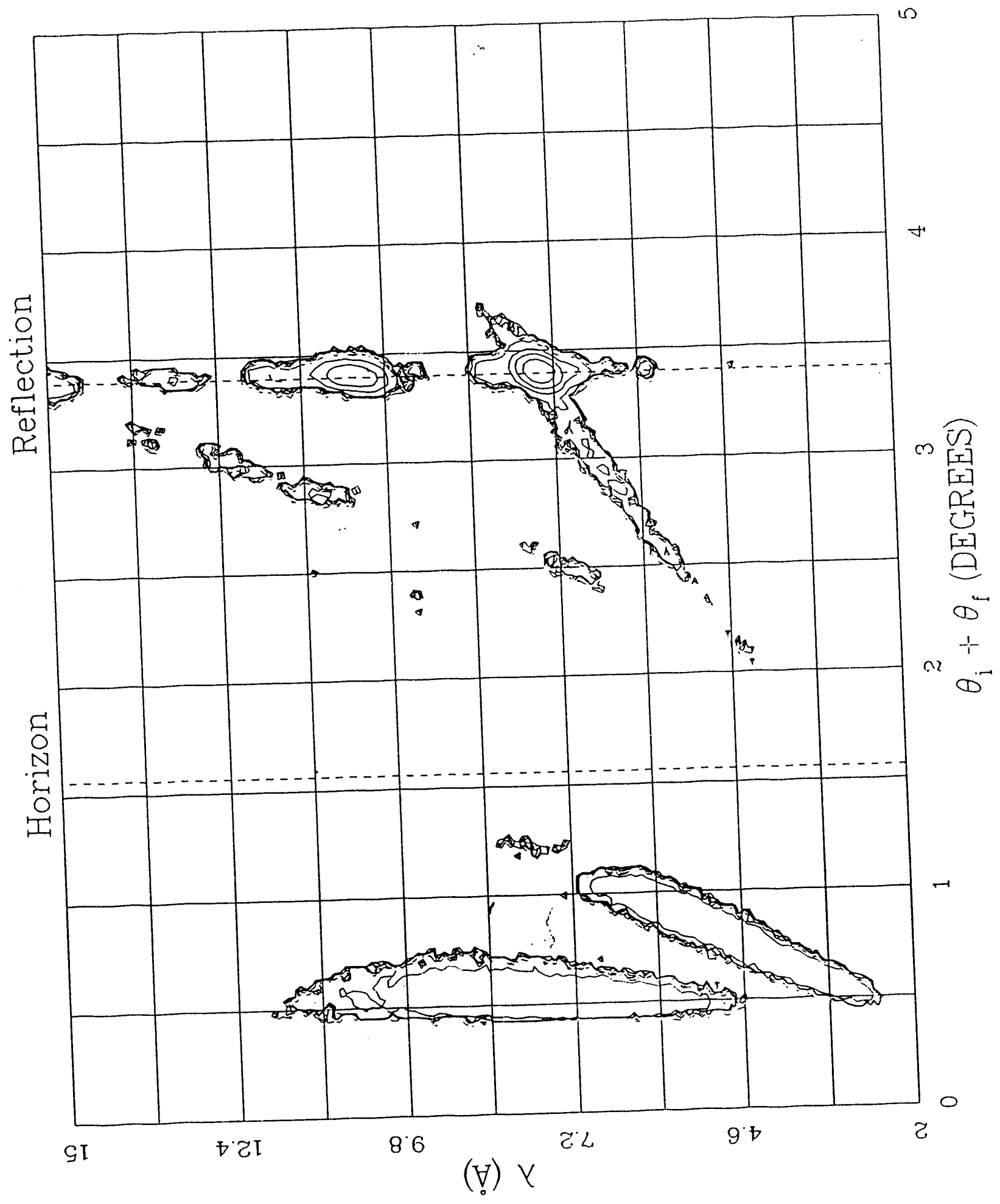




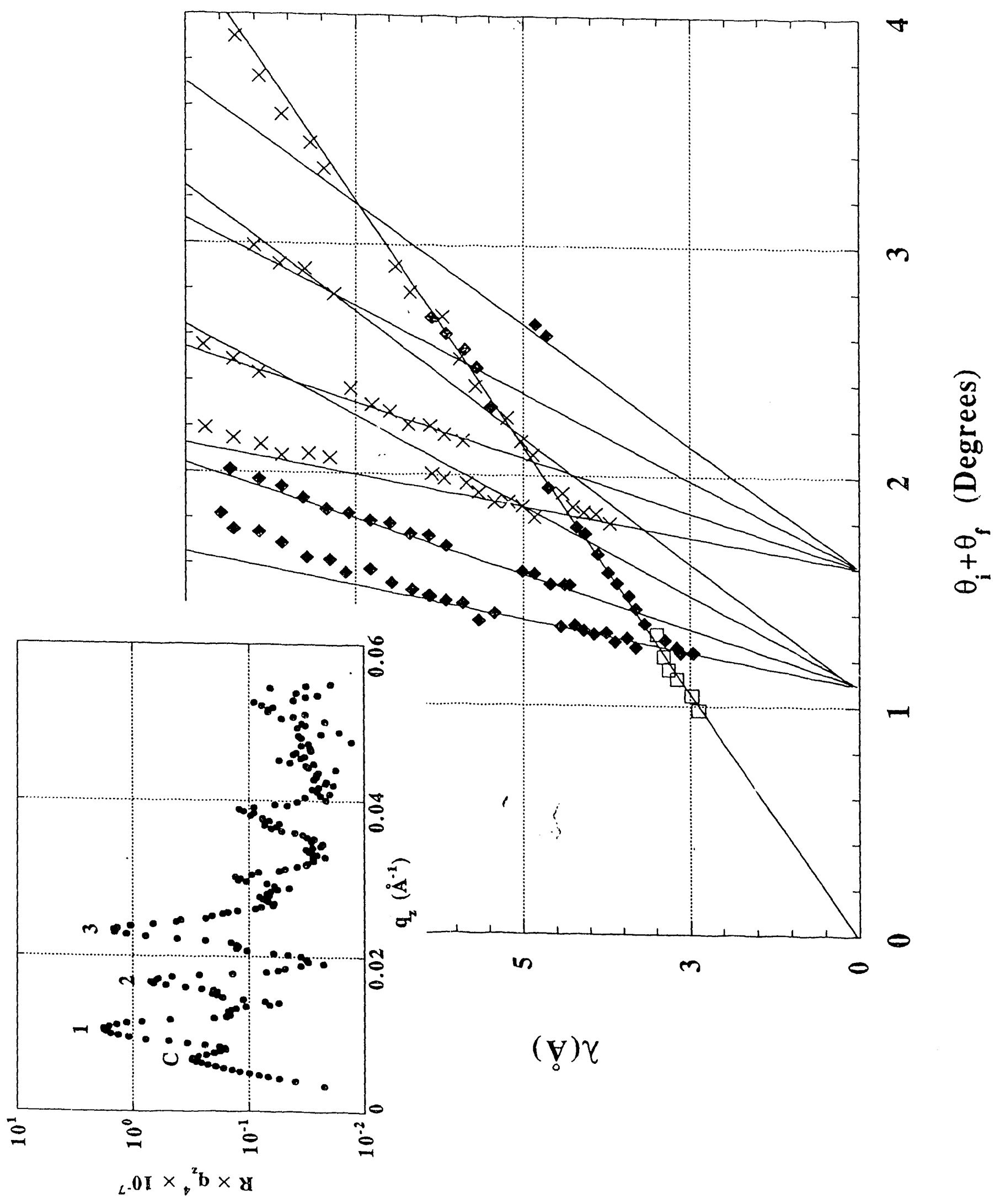




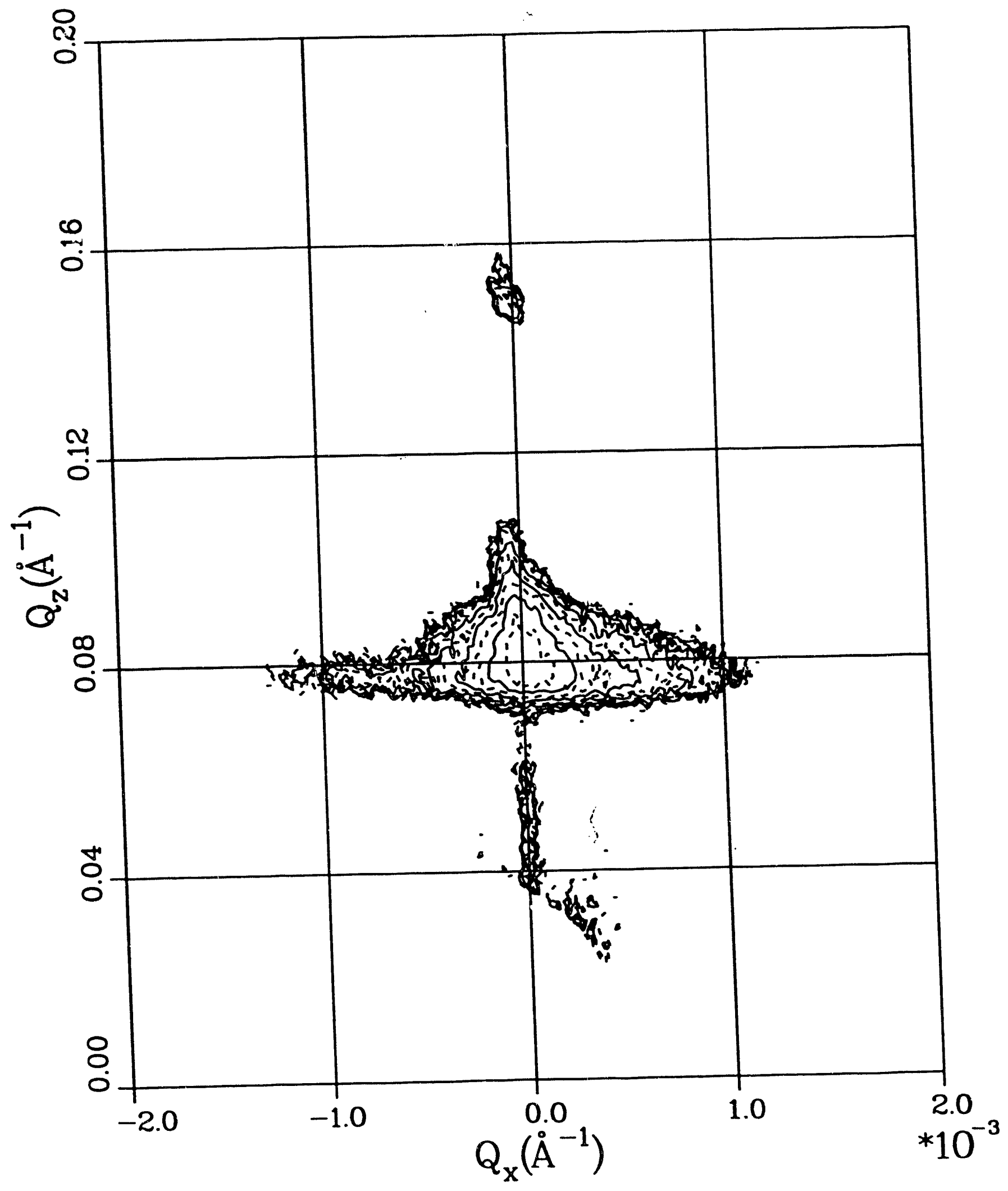



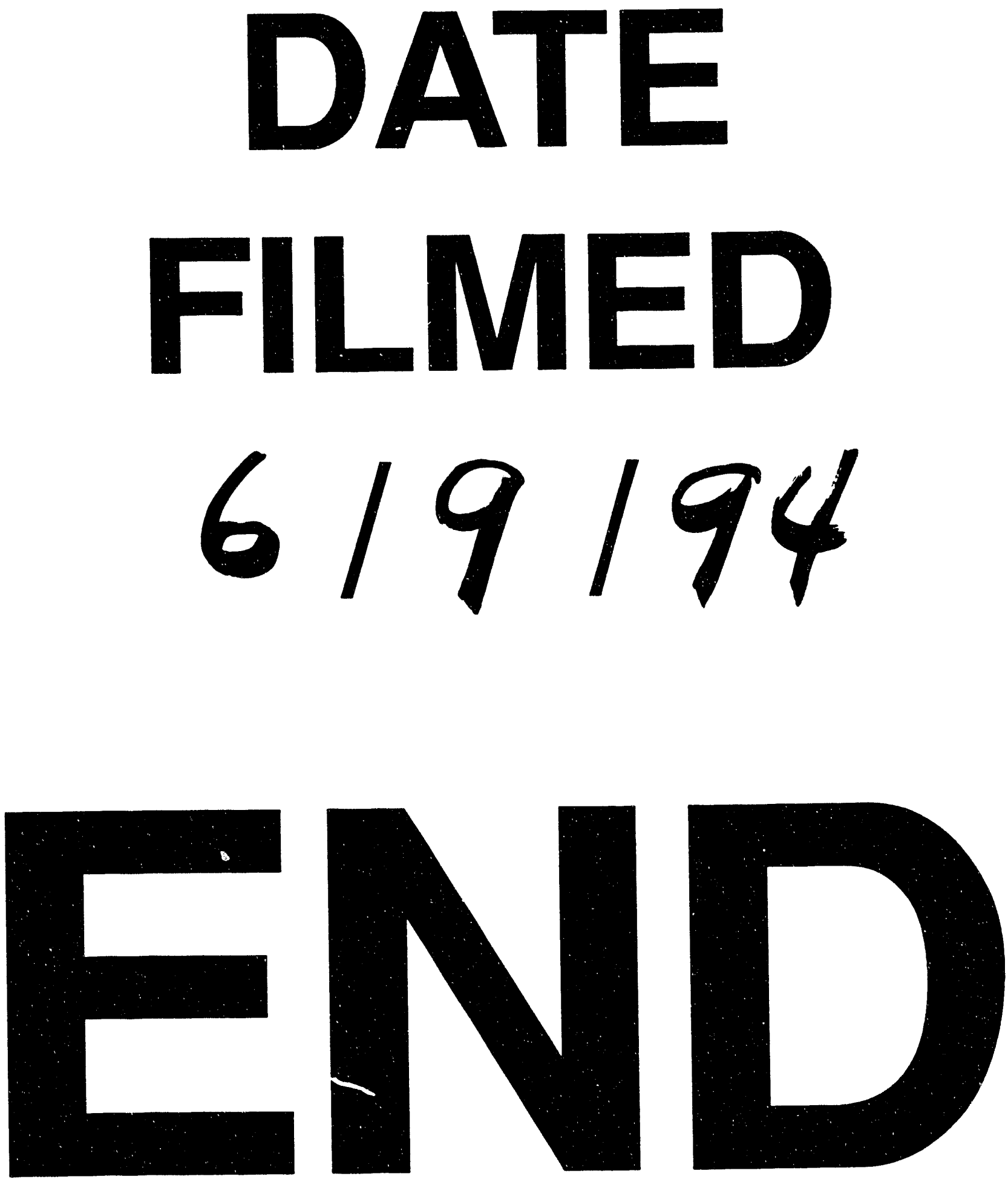
